\title{
New Hybrid Quasi-Newton Algorithms for Large Scale Optimization
}

Abbas Y. Al-Bayati

Sawsan S. Ismail

profabbasalbayati@yahoo.com

College of Computer Sciences and

Mathematics/University of Mosul/Iraq

Received on: 24/4/2005

\section{ABSTRACT}

College of Educations

University of Mosul/Iraq

Accepted on: 2/4/2006

Two new hybrid algorithms have been suggested in this paper, the first one utilizes four formula of self-scaling update matrix was used. The matrix is selected according to Buckley method in each step. The new algorithm has been compared with BFGS standard algorithm by means of (10) multi-dimensional standard functions.

As for the second new hybrid algorithm, a new method is used to test the conjugate coefficient $(\beta)$ which consists of Hestenes Stiefel (HS) and Dai and yuan (DY). Then it is compared with BFGS and PCG algorithms, which uses BFGS update, by means of (10) multi-dimesional standard functions.

Numerical results in general indicates the efficiency of the algorithms proposed in this paper by using this number of non-linear functions in this domain.

Keywords: Large Scale Optimization, Quasi-Newton Algorithms, selfscaling update matrix.

$$
\begin{aligned}
& \text { خوارزميات هجينية لخوارزمية شبيهة نيوتن في الأمثلية ذات القياس الواسع }
\end{aligned}
$$

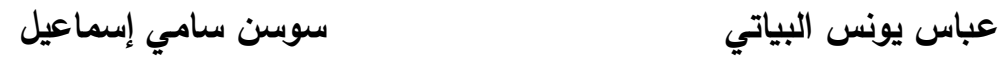

$$
\begin{aligned}
& \text { كلية علوم الحاسبات والرياضيات، جامعة الموصل كلية التربية، جامعة الموصل }
\end{aligned}
$$

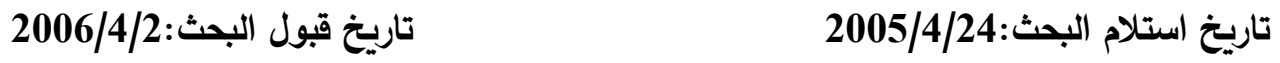

$$
\begin{aligned}
& \text { الملخص } \\
& \text { في هذا البحث تم استحداث خوارزميتين هجينتين جديدتين، الأولى نستخدم فيها أربع صيخ }
\end{aligned}
$$

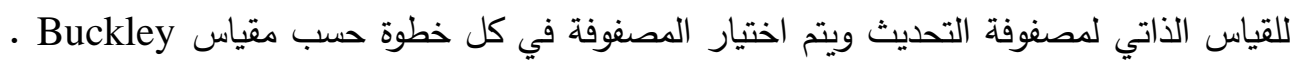

$$
\begin{aligned}
& \text { وتمت مقارنتها مع خوارزمية BFGS القياسية وباستعمال (10) دوال قياسية من ذوات الأبعاد } \\
& \text { المختلفة. } \\
& \text { والخوارزمية الهجينية الجديدة الثانية نستخدم فيها طريقة جديدة لاختبار معامل الترافق }
\end{aligned}
$$

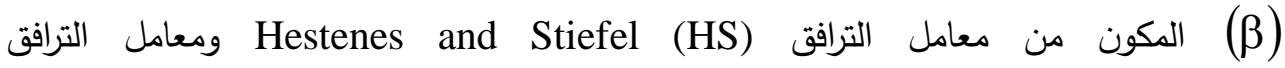

$$
\begin{aligned}
& \text { وتمت مقارنتها مع خوارزميتي DFGS و DCG و PCG and Yuan (DY) } \\
& \text { قياسية من ذوات الأبعاد المختلفة. }
\end{aligned}
$$


النتائج الحسابية بثكل إجمالي تثير إلى كفاءة الخوارزميات المقترحة في هذا البحث وباستعمال هذا العدد من الدوال اللاخطية في هذا المجال.

الكلمات المفتاحية: الأمثلية ذات القياس الواسع، خوارزمية شبيه نيوتن، القياس الذاتي لمصفوفة التحديث.

1. مقدمة

إن موضوع الأمثلية قديم قدم التاريخ ولكن أخذ وضعه الثابت والمفيد بعد تطور العلوم وتقدم البشرية على يد بعض كبار العلماء. ولقد اقتصر بحثنا على التركيز في حقل الأمثلية غير المقيدة التي لا يرتبط حلها بقيود جانبية تؤخذ من الوسط والمؤثرات المحيطة بهذه المشكلة. ويضمن لهن

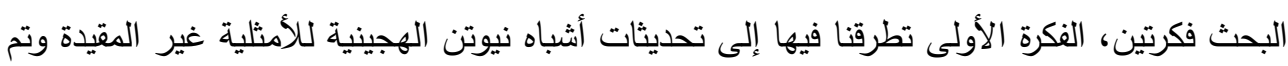

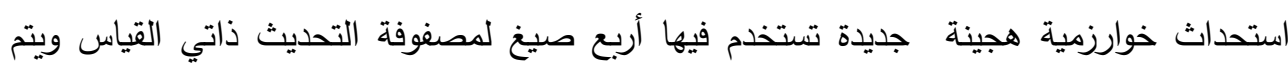
اختيار المصفوفة في كل خطوة حسب مقياس بكلي، وتمت مقارنتها مع خوارزمية BFGS القياسية.

الفكرة الثانية تطرقنا فيها إلى شرح بعض خوارزميات التدرج المترافق الهجينية الكفوةة، وتم استحداث خوارزمية هجينة جديدة تستخدم فيها طريقة جديدة لاختبار معامل الترافق م الناتج من

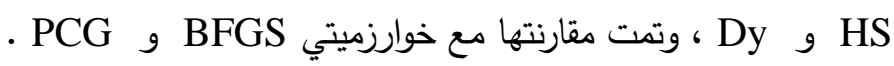

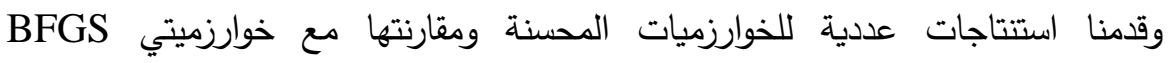
القياسية و PCG لدوال غير خطية وفي أبعاد مختلفة. كما تم اقتراح عمل مستقبلي خاص بهذات

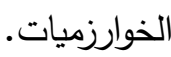

\section{2. الخوارزميات المقترحة}

بالنسبة للخوارزمية الهجينية الأولى تستخدم فيها أربع صيغ القياس الذاتي لمصفوفة

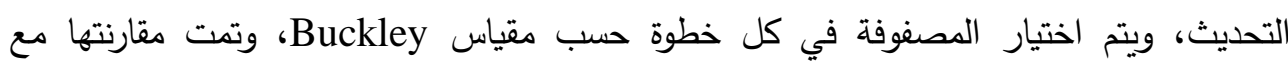
خوارزمية BFGS القياسية.

$$
\text { وكانت صيغ مصفوفات التحديث المستعملة كالآتي: }
$$

2.1 تصديثات المتري المتغير ذات القياس الذاتي من عائلة Oren $H_{k+1}=\left[H_{k}-\frac{H_{k} y_{k} y_{k}^{T} H_{k}^{T}}{y_{k}^{T} H_{k} y_{k}}+w_{k} w_{k}^{T}\right] \zeta_{k}+\frac{v_{k} v_{k}^{T}}{v_{k}^{T} y_{k}}$

$\mathrm{w}_{\mathrm{k}}=\left(\mathrm{y}_{\mathrm{k}}^{\mathrm{T}} \mathrm{H}_{\mathrm{k}} \mathrm{y}_{\mathrm{k}}\right) \mathrm{v}_{\mathrm{k}}-\left(\mathrm{v}_{\mathrm{k}}^{\mathrm{T}} \mathrm{y}_{\mathrm{k}}\right) \mathrm{H}_{\mathrm{k}} \mathrm{y}_{\mathrm{k}}$ 


$$
\zeta_{\mathrm{k}}=\frac{\mathrm{y}_{\mathrm{k}}^{\mathrm{T}} \mathrm{v}_{\mathrm{k}}}{\mathrm{v}_{\mathrm{k}}^{\mathrm{T}} \mathrm{y}_{\mathrm{k}}}
$$

$$
\text { حيث أن C هو مقياس ذاتي مأخوذ من شرط }
$$

2.2 تحديث المتري المتغير ذات القياس الذاتي (Al-Bayati, 1991) $\mathrm{H}_{\mathrm{k}+1}=\mathrm{H}_{\mathrm{k}}-\frac{\mathrm{H}_{\mathrm{k}} \mathrm{y}_{\mathrm{k}} \mathrm{y}_{\mathrm{k}}^{\mathrm{T}} \mathrm{H}_{\mathrm{k}}}{\mathrm{y}_{\mathrm{k}}^{\mathrm{T}} \mathrm{H}_{\mathrm{k}} \mathrm{y}_{\mathrm{k}}}+w_{\mathrm{k}} \mathrm{w}_{\mathrm{k}}^{\mathrm{T}}+\sigma_{\mathrm{k}}\left(\frac{\mathrm{v}_{\mathrm{k}} \mathrm{v}_{\mathrm{k}}^{\mathrm{T}}}{\mathrm{v}_{\mathrm{k}} \mathrm{y}_{\mathrm{k}}}\right)$

$\sigma_{k}=\frac{1}{\zeta_{\mathrm{k}}}$

2.3 صيغة تحديث للمتري المتغير (Al-Maha, 1995) ذات القياس الذاتي

$\mathrm{H}_{\mathrm{k}+1}=\mathrm{H}_{\mathrm{k}}+\frac{\left(\mathrm{sc}_{1} * \mathrm{v}_{\mathrm{k}}-\mathrm{H}_{\mathrm{k}} \mathrm{y}_{\mathrm{k}}\right) \mathrm{v}_{\mathrm{k}}^{\mathrm{T}}}{\mathrm{v}_{\mathrm{k}}^{\mathrm{T}} \mathrm{y}_{\mathrm{k}}}$

$$
\rho_{\mathrm{k}}=\mathrm{sc}_{1}=\frac{\mathrm{y}_{\mathrm{k}}^{\mathrm{T}} \mathrm{y}_{\mathrm{k}}}{\mathrm{v}_{\mathrm{k}}^{\mathrm{T}} \mathrm{y}_{\mathrm{k}}} \text { هيث أن المقياس الذاتي هو }
$$

2.4

$$
\mathrm{H}_{\mathrm{k}+1}=\mathrm{H}_{\mathrm{k}}-\frac{\mathrm{H}_{\mathrm{k}} \mathrm{y}_{\mathrm{k}} \mathrm{v}_{\mathrm{k}}^{\mathrm{T}}+\mathrm{v}_{\mathrm{k}} \mathrm{y}_{\mathrm{k}}^{\mathrm{T}} \mathrm{H}_{\mathrm{k}}}{\mathrm{v}_{\mathrm{k}}^{\mathrm{T}} \mathrm{y}_{\mathrm{k}}}+\left(1+\frac{\mathrm{y}_{\mathrm{k}}^{\mathrm{T}} \mathrm{H}_{\mathrm{k}} \mathrm{y}_{\mathrm{k}}}{\mathrm{v}_{\mathrm{k}}^{\mathrm{T}} \mathrm{y}_{\mathrm{k}}}\right)\left(\frac{\mathrm{v}_{\mathrm{k}} \mathrm{v}_{\mathrm{k}}^{\mathrm{T}}}{\mathrm{v}_{\mathrm{k}}^{\mathrm{T}} \mathrm{y}_{\mathrm{k}}}\right)
$$

3. خوارزميات التدرج المترافق المشروط مسبقاً

\section{Preconditioned Conjugate Gradient Algorithms (PCG)}

إن كلاً من خوارزميات أشباه نيوتن (QN) وخوارزميات التدرج المترافق (CG) لها مزايا

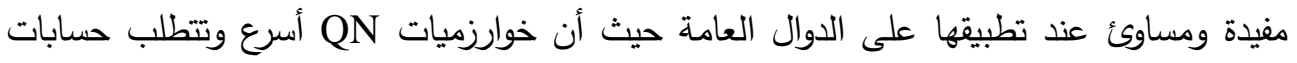

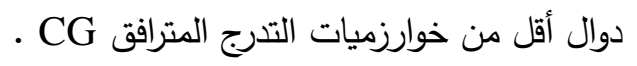

كذلك خوارزمية QN تكوّن متتابعة من مصفوفات متتاظرة وموجبة قطعاً بصورة اعتيادية. والخوارزميات المترية لا يكون استعمالها فعالاً بسبب الخزن ولهذا تم التطرق إلى صنف جدئ جديد من

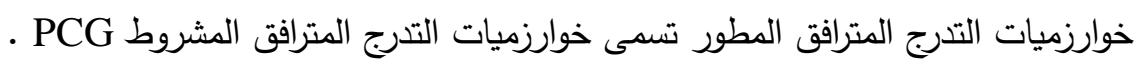

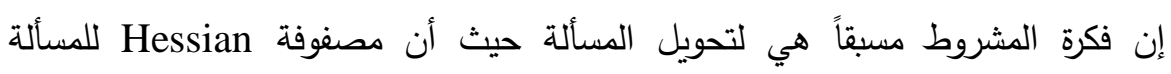
المتحولة تملك القيم المميزة وتكون حسنة الشرط. الغرض من خوارزمية PCG هو لحفظ متطلبات

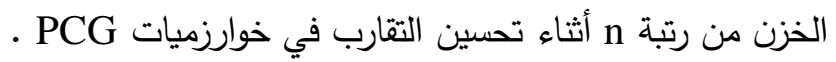


خوارزمية CG القياسية ليست فعالة دائماً لكن الاشتراط يفرض استعمال المصفوفة المناسبة التي تعجل التقارب في خوارزمية CG عن طريق تحويل المتغيرات بينما يحافظ على الخواص الأساسية لهذه الخوارزمية. المصفوفات المشروطة أسست على تقريبات معكوس Hessian

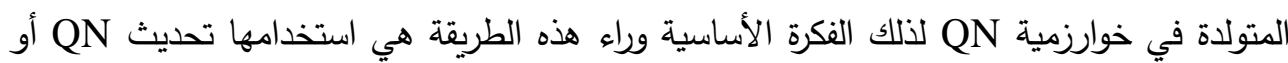
VM للتعجيل بخوارزمية التدرج المترافق. PCG صفات عامة لخوارزمية 1. تحتاج إلى خزن قليل و إلى زمن حسابات قليل. 2. ليست حساسة جداً فيما يتعلق بدقة خط البحث. 3. تملك خاصية التوقف التربيعي.

4. تحديثات أشباه نيوتن الهجينية (New I)

\section{Hybrid Quasi-Newton Update}

نقدم في هذه الخوارزمية التحديثات المتعددة لطريقة أشباه نيوتن الهجينية لمسائل الأمثلية

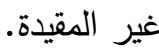
هذه الخوارزميات تولد عدة تحديثات أشباه نيوتن QN عند كل تكرار نقوم باستعراض فئة

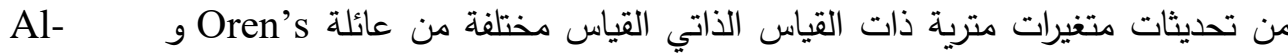
- Al-Maha g Bayati

إن محور اهتمامنا هو التوسع الهجين لطريقة أشباه نيوتن والذي سيلائم الحل لمسائل

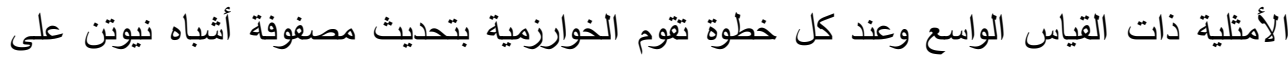
طول n غير معتمدة على الاتجاهات وذلك بتقييم قيم الدالة بثكل موازي والتدرج عند n من النقاط. ولهذا السبب يعتبر هذه الخوارزمية هي خوارزمية هجينية لطريقة أثباه نيوتن بحيث عندما تطبق هذه الخوارزميات لحل المسائل العملية ستكون الآلية التسلسلية والموازية قادرة على كثف هائف الأشكال المختلفة لتحديثات أشباه نيوتن خلاه عملية التصفير .

\section{New 1 Algorithm الخوارزمية الجديدة الأولى 4.1}

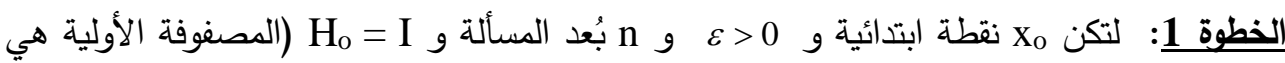

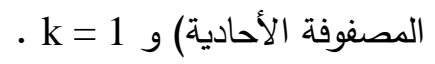

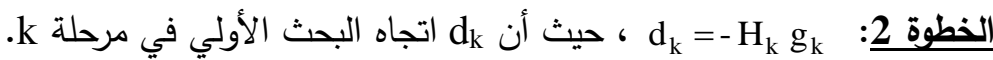




$$
\begin{aligned}
& \text { الخطوة 3: نصغر الدالة } \\
& \text { من خلال إجراءات البحث الخطي. } \\
& \mathrm{x}_{\mathrm{k}+1}=\mathrm{x}_{\mathrm{K}}+\lambda_{\mathrm{k}} \mathrm{d}_{\mathrm{k}} \\
& \text { ج }
\end{aligned}
$$

الخطوة 4: احسب التقارب إذا حقق ع >|| الغ

الخطوة 5: استخدام $y_{k}=g_{k+1}-g_{k}, v_{k}=x_{k+1}-x_{k}$ حيث

$$
\begin{aligned}
& \text { الخطوة 6: } \\
& \text { II = II + } 1 \text { : الخطوة }
\end{aligned}
$$

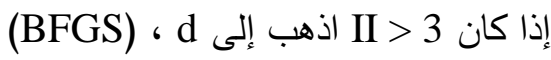

$$
\text { بعكسه اذهب إلى (a , (a ) }
$$

(Al-Bayati) ذاتي القياس (a

Hاتي القياس (Oren (b

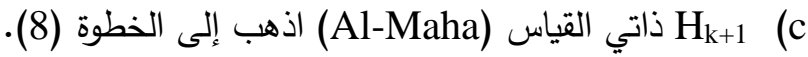

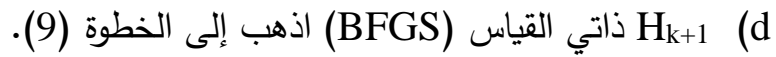

$$
\text { الخطوة 8: استخدم مقياس الاسترجاع التالي: }
$$

إذا كان $\left|\frac{g_{k}^{\mathrm{T}} \mathrm{H}_{\mathrm{k}+1} \mathrm{~g}_{\mathrm{k}+1}}{\mathrm{~g}_{\mathrm{k}+1}^{\mathrm{T}} \mathrm{H}_{\mathrm{k}+1} \mathrm{~g}_{\mathrm{k}+1}}\right|>0.01$ اذهب إلى الخطوة (9). وإلا اذهب إلى الخطوة

$$
\begin{aligned}
& \beta^{H S}=\frac{\mathrm{g}_{\mathrm{k}+1}^{\mathrm{T}} \mathrm{Hy}_{\mathrm{k}}}{\mathrm{y}_{\mathrm{k}}^{\mathrm{T}} \mathrm{d}_{\mathrm{k}}} \\
& \text { الخطوة 10: احسب مقياس استرجاع إذا كانت } \\
& \left\|g_{k+1}\right\|<-0.8 \mathrm{~g}_{\mathrm{k}+1}^{\mathrm{T}} \mathrm{d}_{\mathrm{k}} \\
& \text { و k=n اذهب إلى الخطوة (1). } \\
& \text { وإلا k= k } 1 \text { k واذهب إلى الخطوة (3). }
\end{aligned}
$$

5. الخوارزمية الفاعلة الهجينية المشروطة مسبقاً للتدرج المترافق

\section{An Efficient Hybrid Preconditioning Conjugate Gradient Algorithm}

تم في هذه الفقرة استحداث خوارزمية هجينية تستخدم فيها طريقة جديدة لاختبار معامل

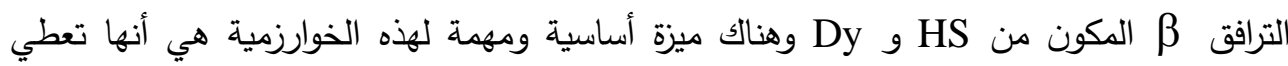


اتجاهاً للبحث منحدراً في كل تكرار وتتقارب بشكل شامل شريطة أن يحقق البحث شروط هolfe

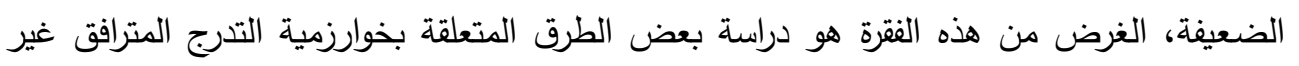
الخطي الهجينية الجديدة، وإيجاد خوارزميات كفوءة من بينها. لاحظ مشكلة الأمثلية غير المقيدة التالية

$\min _{\mathrm{x}} \mathrm{F}(\mathrm{x}) \quad, \quad \mathrm{x} \in \mathrm{R}$

حيث أن F دالة مستقيمة (smooth) ومشتقتها متوفرة. إن طرق التدرج المترافق تعد فاعلة جداً وكفوءة لحل (1) خاصة عندما يكون بُعد الدالة كبيراً وله الصيغة الآتية: $\mathrm{x}_{\mathrm{k}+1}=\mathrm{x}_{\mathrm{k}}+\lambda_{\mathrm{k}} \mathrm{d}_{\mathrm{k}}$

$d_{k}=\left\{\begin{array}{ll}-g_{k} & \text { For } k=1 \\ -g_{k}+\beta_{k} d_{k-1} & \text { For } k \geq 2\end{array}\right\}$

عند تحليلات التقارب وتطبيق خوارزمية التدرج المترافق فان إحداها غالباً ما يستلزم أن يكون البحث الخطي مضبوطاً أو يحقق شروط Wolfe القوية وتحديداً: $\mathrm{F}\left(\mathrm{x}_{\mathrm{k}}\right)-\mathrm{F}\left(\mathrm{x}_{\mathrm{k}}+\lambda_{\mathrm{k}} \mathrm{d}_{\mathrm{k}}\right) \geq-\delta \lambda_{\mathrm{k}} \mathrm{g}_{\mathrm{k}}^{\mathrm{T}} \mathrm{d}_{\mathrm{k}}$

$$
\left|g\left(x_{k}+\lambda_{k} d_{k}\right)^{T} d_{k}\right| \leq-\sigma g_{k}^{T} d_{k}
$$

$$
\text { حيث أن } 0<\delta<0<0
$$

بالنسبة للأخير نسمي البحث الخطي على أنه البحث الخطي القوي لـ لـ Wolfe عند أخذنا

$$
\text { : لجِ }
$$

$\beta_{k}^{D y}=\frac{\left\|\mathrm{g}_{\mathrm{k}}\right\|^{2}}{\mathrm{~d}_{\mathrm{k}-1}^{\mathrm{T}} \mathrm{y}_{\mathrm{k}-1}}$

وقد ثبت أن قيمة م في هذه الطريقة تخلق اتجاهاً منحدراً عند كل تكرار وتتقارب بشكل

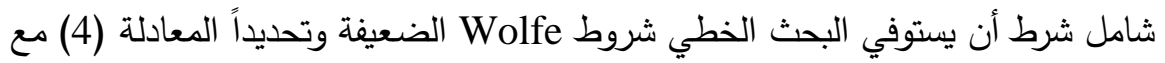
$\mathrm{g}\left(\mathrm{x}_{\mathrm{k}}+\lambda_{\mathrm{k}} \mathrm{d}_{\mathrm{k}}\right)^{\mathrm{T}} \mathrm{d}_{\mathrm{k}} \geq \sigma \mathrm{g}_{\mathrm{k}}^{\mathrm{T}} \mathrm{d}_{\mathrm{k}}$

في هذه الحالة نسي البحث الخطي بأنه البحث الخطي الضعيف لـ Wolfe .

5.1

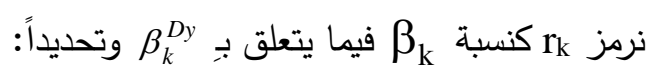

$\mathrm{r}_{\mathrm{k}}=\frac{\beta_{k}}{\beta_{k}^{D y}}$ 
حيث ثبت أن الخوازرمية (2) و (3) مع البحث الخطي الضعيف لـ لـ Wolfe تخلق اتجاه

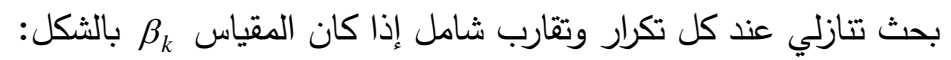

$-\mathrm{c}<\mathrm{r}_{\mathrm{k}}<1$

$$
\text { c }=\frac{1-\sigma}{1+\sigma}>0 \text { حيث أن }
$$

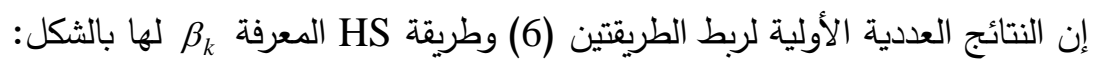

$$
\beta_{k}^{H S}=\frac{g_{k}^{T} \mathrm{y}_{\mathrm{k}-1}}{d_{k-1}^{T} \mathrm{y}_{\mathrm{k}-1}}
$$

تظهر أن الخوارزميتين الهجينتين التاليتين اداؤها أفضل من طريقة PR

الخوارزمية الأولى تعرف بالثكل

$$
\beta_{\mathrm{k}}=\max \left\{-\mathrm{c} \beta_{\mathrm{k}}^{\mathrm{Dy}}, \min \left\{\beta_{\mathrm{k}}^{\mathrm{HS}}, \beta_{\mathrm{k}}^{\mathrm{Dy}}\right\}\right\}
$$

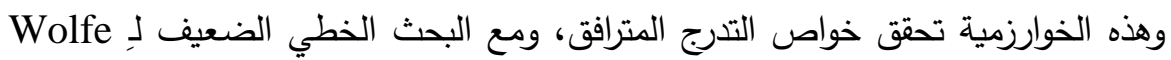
تخلق اتجاهاً تتازلياً في كل تكرار وتتقارب بشكل شامل.

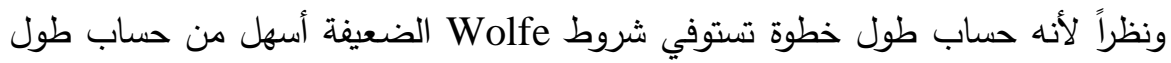
خطوة تستوفي شروط Wolfe القوية فعند اختبارنا للخوارزمية (11) مع البحث الخطي الضعيف لـ لـ

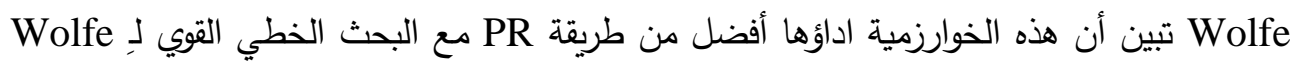

$$
\beta_{\mathrm{k}}=\max \left\{0, \min \left\{\beta_{\mathrm{k}}^{\mathrm{HS}}, \beta_{\mathrm{k}}^{\mathrm{Dy}}\right\}\right\} \quad \text { الخوارزمية الثانية المقترحة بالثكل }
$$

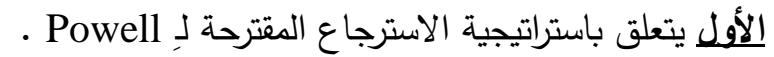

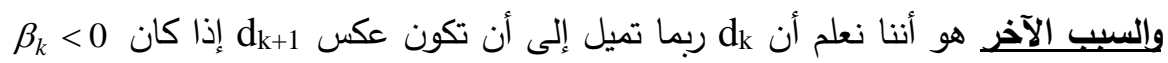
• وهكذا فان التحديد $\beta_{k} \geq 0$ سوف يمنع اتجاهي البحث المتتابعين من أن يكونا متعاكسين. وقد أظهرت نتائجنا العددية أن الطريقة الهجينية (12) ذات اداء أفضل من الطريقة الهجينية (11).

\section{The Out Lines of the New PCG Algorithm (New II)}

5.2 خطوات لخوارزمية PCG الجديدة (New II)

الخطوة (1): لتكن X نقطة بداية و

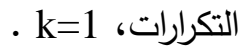

$$
\begin{aligned}
& \text { الخطوة (2): اتجاه البحث الخطي d d }
\end{aligned}
$$




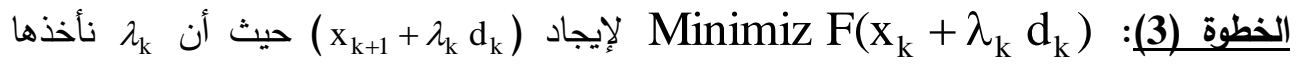

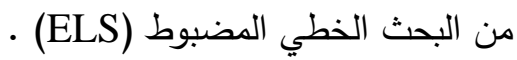

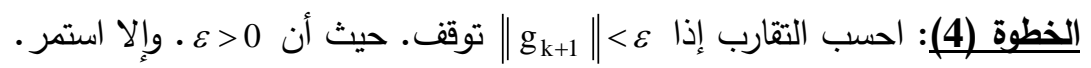
الخطوة (5): احسب

$\mathrm{v}_{\mathrm{k}}=\mathrm{x}_{\mathrm{k}+1}-\mathrm{x}_{\mathrm{k}}$

$\mathrm{y}_{\mathrm{k}}=\mathrm{g}_{\mathrm{k}+1}-g_{\mathrm{k}}$

الخطوة (6): قم بتحديث H بإضافة مصفوفة تصحيحية E للحصول على H $\mathrm{H}_{\mathrm{k}+1}=\mathrm{H}_{\mathrm{k}}+E_{k}$ حيث أن Ek هي مصفوفة تصحيحية تحقق شرط QN والذي هو $\mathrm{H}_{\mathrm{k}+1} \mathrm{y}_{\mathrm{k}}=\rho_{\mathrm{k}} \mathrm{v}_{\mathrm{k}}$

$\rho_{\mathrm{k}}=\frac{\mathrm{y}_{\mathrm{k}}^{\mathrm{T}} \mathrm{H}_{\mathrm{k}} \mathrm{y}_{\mathrm{k}}}{\mathrm{y}_{\mathrm{k}}^{\mathrm{T}} \mathrm{v}_{\mathrm{k}}}$ حيث أن

$$
\mathrm{y}_{\mathrm{k}}^{\mathrm{T}} \mathrm{v}_{\mathrm{k}}
$$

وأن

$\mathrm{H}_{\mathrm{k}+1}=\underset{\text { Al-Bayati }}{\operatorname{Update}}\left(\mathrm{H}_{\mathrm{k}} \mathrm{y}_{\mathrm{k}} \mathrm{v}_{\mathrm{k}}\right)$

الخطوة (7):

$c=\frac{1-0.1}{1+0.1}$

$\beta^{\mathrm{HS}}=\frac{\mathrm{y}_{\mathrm{k}}^{\mathrm{T}} \mathrm{H}_{\mathrm{k}+1} \mathrm{~g}_{\mathrm{k}+1}}{\mathrm{~d}_{\mathrm{k}}^{\mathrm{T}} \mathrm{y}_{\mathrm{k}}}$

$\beta^{\text {Dy }}=\frac{\mathrm{g}_{\mathrm{k}+1}^{\mathrm{T}} \mathrm{H}_{\mathrm{k}+1} \mathrm{~g}_{\mathrm{k}+1}}{\mathrm{~d}_{\mathrm{k}}^{\mathrm{T}} \mathrm{y}_{\mathrm{k}}}$

(الخطوة (8):

$\operatorname{Beta}=\operatorname{Max}\left(0, \operatorname{Min}\left(\beta^{\mathrm{HS}}, \beta^{\text {Dy }}\right)\right)$

$\operatorname{Beta}=\operatorname{Max}\left(-\mathrm{c} \cdot \beta^{\text {Dy }}, \operatorname{Min}\left(\beta^{\mathrm{HS}}, \beta^{\text {Dy }}\right)\right)$

$\mathrm{d}_{\mathrm{k}+1}=-\mathrm{H}_{\mathrm{k}+1} \mathrm{~g}_{\mathrm{k}+1}+\beta_{\mathrm{k}} \mathrm{d}_{\mathrm{k}}$

(الخطوة (9)

الخطوة (10): إذا كان

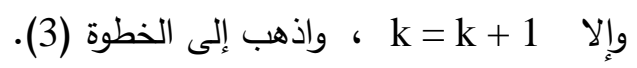


6. بعض خواص خوارزميتي New I و New II 1. للدالة التربيعية مع خط بحث غير مضبوط.

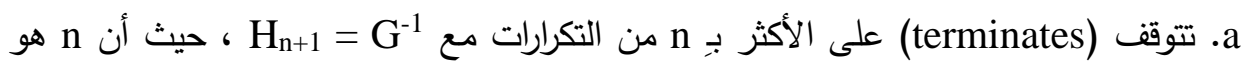

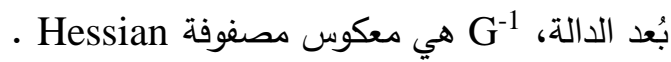

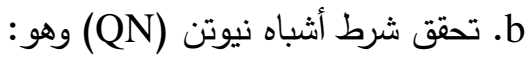

$$
\begin{aligned}
& \mathrm{H}_{\mathrm{k}+1} \mathrm{y}_{\mathrm{k}}=\mathrm{v}_{\mathrm{k}} \\
& \mathrm{v}_{\mathrm{k}}=\mathrm{x}_{\mathrm{k}+1}-\mathrm{x}_{\mathrm{k}} \\
& \mathrm{y}_{\mathrm{k}}=\mathrm{g}_{\mathrm{k}+1}-\mathrm{g}_{\mathrm{k}}
\end{aligned}
$$

$$
\text { حيث أن }
$$

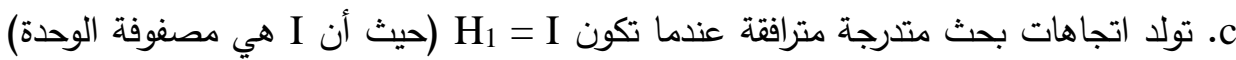

$$
\begin{aligned}
& \text { و k =n (حيث أن k تساوي عدد التكرارات و n بُعد الدالة). } \\
& \text { 2. للدوال العامة }
\end{aligned}
$$

a aحافظ على المصفوفة الموجبة قطعاً H مبهذا تبقي على خاصية الانحدار.

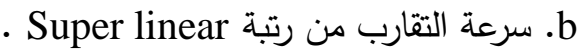

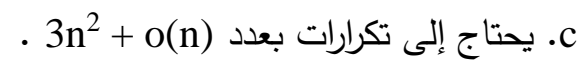

dd تقارب شامل إذا كانت الدالة محدبة بصرامة (strictly convex)، (مع بحث خطي غير

$$
\text { مضبوط). }
$$

7. - النتائج الحسابية والاستنتاجات

\section{Numerical Results and Conclusions}

\section{1}

اختبارات المقارنة تتضمن 10 دوال اختبار معطاة بشكل واضح في الملحق مع أبعاد مختلفة، كل النتائج تم الحصول عليها باستخدام الدقة المضاعفة على حاسبة من نوع • وباستخدام برامج مكتوبة بلغة فورتران Pentium Computer أدوات تقييم هذه الخوارزميات ومقارنتها معتمدة على العدد الكلي للتكرارات (NOI) والعدد الكلي للدوال المحسوبة (NOF). خط البحث المستعمل يعتمد على التقنية التكعيبية ودالة الهدف ونقطة البداية الأولية قد تم إعطاؤها لكل مسألة اختبار . وقد تم استخدام مقياس الاسترجاع داعل ع(x) نفسه مع الخوارزميات الأصلية والجديدة. 
عباس يونس البباتي وسوسن سامي إسعاعيل

ومقياس التوقف المستخدم لإيقاف الخوارزمية لجميع الحالات في الطريقة New I و

هو New II

$\left\|\mathrm{g}_{k+1}\right\| \leq 1 \times 10^{-5}$

الجدول (1): الأداء المقارن لجميع خوارزميات (طريقة BFGS القياسية والخوارزمية

(NEW I للمجموعة الاختبارية من الدول المستعملة في الجدول وللأبعاد

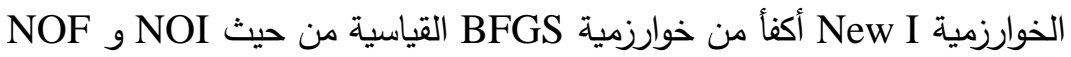

$n=4$

الجدول (1 A):

\begin{tabular}{|c|c|c|c|c|c|c|c|c|}
\hline \multirow{2}{*}{ Test Function } & \multicolumn{9}{|c|}{ New I } & \multicolumn{2}{c|}{ Original BFGS } \\
\cline { 2 - 10 } & NOI & NOF & nou1 & nou2 & nou3 & nou4 & NOI & NOF \\
\hline \hline 1 & 18 & 43 & 17 & 1 & 2 & 0 & 31 & 100 \\
\hline 2 & 10 & 30 & 9 & 1 & 2 & 1 & 17 & 48 \\
\hline 3 & 6 & 20 & 5 & 5 & 0 & 4 & 9 & 23 \\
\hline 4 & 17 & 43 & 16 & 0 & 2 & 0 & 28 & 65 \\
\hline 5 & 10 & 49 & 9 & 1 & 2 & 1 & 30 & 156 \\
\hline 6 & 26 & 61 & 25 & 0 & 0 & 0 & 38 & 102 \\
\hline 7 & 22 & 58 & 21 & 2 & 3 & 1 & 26 & 57 \\
\hline 8 & 10 & 29 & 9 & 0 & 3 & 0 & 14 & 30 \\
\hline 9 & 19 & 49 & 18 & 1 & 1 & 1 & 33 & 80 \\
\hline 10 & 8 & 20 & 7 & 0 & 1 & 0 & 10 & 26 \\
\hline \hline Total & $\mathbf{1 4 6}$ & $\mathbf{4 0 2}$ & $\mathbf{1 3 6}$ & $\mathbf{1 1}$ & $\mathbf{1 6}$ & $\mathbf{8}$ & $\mathbf{2 3 6}$ & $\mathbf{6 8 7}$ \\
\hline
\end{tabular}

$\mathrm{n}=\mathbf{5 0 0}$

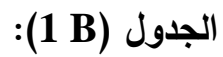

\begin{tabular}{|c|c|c|c|c|c|c|c|c|}
\hline \multirow{2}{*}{ Test Function } & \multicolumn{9}{|c|}{ New I } & \multicolumn{2}{c|}{ Original BFGS } \\
\cline { 2 - 10 } & NOI & NOF & nou1 & nou2 & nou3 & nou4 & NOI & NOF \\
\hline \hline 1 & 19 & 50 & 18 & 2 & 3 & 0 & 40 & 142 \\
\hline 2 & 11 & 32 & 10 & 1 & 3 & 1 & 18 & 50 \\
\hline 3 & 6 & 20 & 5 & 5 & 0 & 4 & 9 & 23 \\
\hline 4 & 24 & 59 & 23 & 1 & 3 & 1 & 36 & 82 \\
\hline 5 & 13 & 79 & 12 & 2 & 3 & 1 & 22 & 100 \\
\hline 6 & 39 & 99 & 38 & 0 & 0 & 0 & 44 & 133 \\
\hline 7 & 23 & 60 & 22 & 2 & 4 & 1 & 27 & 60 \\
\hline 8 & 19 & 59 & 18 & 1 & 1 & 1 & 25 & 75 \\
\hline 9 & 20 & 74 & 19 & 2 & 2 & 1 & 34 & 83
\end{tabular}




\begin{tabular}{||c|c|c|c|c|c|c|c|c||}
\hline 10 & 9 & 25 & 8 & 1 & 2 & 0 & 10 & 26 \\
\hline \hline Total & $\mathbf{1 8 3}$ & $\mathbf{5 5 7}$ & $\mathbf{1 7 3}$ & $\mathbf{1 7}$ & $\mathbf{2 1}$ & $\mathbf{1 0}$ & $\mathbf{2 6 5}$ & $\mathbf{7 7 4}$ \\
\hline
\end{tabular}

$$
\text { وأظهرت النتائج العددية ان الخوارزميات الجديدة هي كفوءة جداً. }
$$

وكمثال على مدى تحسين New I خوارزمية BFGS كنسبة مئوية وعندما تكون n=4 و و

n=500 نلاحظ الجدولين التاليين : ولينال

\begin{tabular}{|c|c|c|}
\multicolumn{1}{c}{ n=4 } \\
\hline Tools & BFGS & New 1 \\
\hline NOI & $100 \%$ & $61 \%$ \\
\hline NOF & $100 \%$ & $58 \%$ \\
\hline
\end{tabular}

\begin{tabular}{|c|c|c|}
\multicolumn{3}{c}{ n=500 } \\
\hline Tools & BFGS & New 1 \\
\hline NOI & $100 \%$ & $69 \%$ \\
\hline NOF & $100 \%$ & $71 \%$ \\
\hline
\end{tabular}

الجدول (2): يتضمن الجدول نتيجة المقارنة بين طريقة BFGS القياسية و PCG التي تستعمل BFGS والخوارزمية الجديدة (New II (PCG) للمجموعة الاختبارية من الدوال المستعملة

$$
\text { n }
$$

$$
\text { الجدول (2A) }
$$

\begin{tabular}{|c|c|c|c|c|c|c|}
\hline \multirow{2}{*}{$\begin{array}{c}\text { Test } \\
\text { Function }\end{array}$} & \multicolumn{2}{|c|}{ New II PCG } & \multicolumn{2}{c|}{ PCG } & \multicolumn{2}{c|}{ BFGS } \\
\cline { 2 - 7 } & NOI & NOF & NOI & NOF & NOI & NOF \\
\hline \hline 1 & 14 & 39 & 20 & 71 & 22 & 92 \\
\hline 2 & 14 & 37 & 19 & 58 & 19 & 61 \\
\hline 3 & 7 & 18 & 6 & 20 & 8 & 26 \\
\hline 4 & 16 & 49 & 26 & 67 & 24 & 69 \\
\hline 5 & 12 & 51 & 20 & 85 & 18 & 83 \\
\hline 6 & 28 & 80 & 27 & 89 & 26 & 90 \\
\hline 7 & 21 & 53 & 36 & 104 & 37 & 108 \\
\hline 8 & 10 & 22 & 9 & 28 & 9 & 24 \\
\hline 9 & 20 & 58 & 34 & 96 & 33 & 94 \\
\hline 10 & 9 & 23 & 8 & 20 & 8 & 22 \\
\hline \hline Total & $\mathbf{1 5 1}$ & $\mathbf{4 3 0}$ & $\mathbf{2 0 5}$ & $\mathbf{6 3 8}$ & $\mathbf{2 0 4}$ & $\mathbf{6 6 9}$ \\
\hline
\end{tabular}


عباس بيونس البياتسي وسوسن سامي إسماعيل

\begin{tabular}{|c|c|c|c|c|c|c|}
\hline \multirow{2}{*}{$\begin{array}{c}\text { Test } \\
\text { Function }\end{array}$} & \multicolumn{2}{|c|}{ New II PCG } & \multicolumn{2}{c|}{ PCG } & \multicolumn{2}{c|}{ BFGS } \\
\cline { 2 - 7 } & NOI & NOF & NOI & NOF & NOI & NOF \\
\hline \hline 1 & 15 & 41 & 45 & 111 & 51 & 147 \\
\hline 2 & 14 & 37 & 48 & 115 & 49 & 121 \\
\hline 3 & 8 & 21 & 6 & 20 & 8 & 26 \\
\hline 4 & 21 & 59 & 111 & 277 & 61 & 143 \\
\hline 5 & 13 & 65 & 22 & 92 & 19 & 89 \\
\hline 6 & 36 & 102 & 42 & 126 & 42 & 129 \\
\hline 7 & 22 & 55 & 778 & 2113 & 769 & 2238 \\
\hline 8 & 22 & 69 & 34 & 92 & 34 & 96 \\
\hline 9 & 21 & 60 & 625 & 1608 & 610 & 1676 \\
\hline 10 & 9 & 23 & 10 & 25 & 10 & 27 \\
\hline \hline Total & $\mathbf{1 8 1}$ & $\mathbf{5 3 2}$ & $\mathbf{1 7 2 1}$ & $\mathbf{4 5 7 9}$ & $\mathbf{1 6 5 3}$ & $\mathbf{4 6 9 2}$ \\
\hline
\end{tabular}

وكمقارنة لاداء الخوارزمية New II مع خوارزمية BFGS وخوارزمية PCG كنسبة

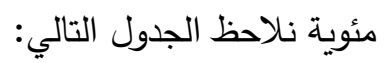

$\mathrm{n}=\mathbf{4}$

\begin{tabular}{|c|c|c|c|c|}
\hline Tools & BFGS & New II & PCG & New II \\
\hline NOI & $100 \%$ & $74 \%$ & $100 \%$ & $73 \%$ \\
\hline NOF & $100 \%$ & $64 \%$ & $100 \%$ & $67 \%$ \\
\hline
\end{tabular}

$\mathrm{n}=\mathbf{5 0 0}$

\begin{tabular}{|c|c|c|c|c|}
\hline Tools & BFGS & New II & PCG & New II \\
\hline NOI & $100 \%$ & $11 \%$ & $100 \%$ & $10 \%$ \\
\hline NOF & $100 \%$ & $11 \%$ & $100 \%$ & $11 \%$ \\
\hline
\end{tabular}

8ppendix الملحق Ap

\section{Generalized Powell Function}

$$
\begin{aligned}
& F(X)=\sum_{i=2}^{n / 4}\left[\left(x_{4 i-3}-10 x_{4 i-2}\right)^{2}+5\left(x_{4 i-1}-x_{4 i}\right)^{2}+\left(x_{4 i-2}-2 x_{4 i-1}\right)^{4}+10\left(X_{4 i-3}-x_{4 i}\right)^{4}\right] \\
& x_{o}=(3,-1,0,1, \ldots)^{T}
\end{aligned}
$$

\section{Generalized Cube Function}

$\mathrm{F}(\mathrm{x})=100\left(\mathrm{x}_{2}-\mathrm{x}_{1}^{3}\right)^{2}+\left(1-\mathrm{x}_{1}\right)^{2}$

$\mathrm{x}_{\mathrm{o}}=(-1.2,1)^{\mathrm{T}}$

\section{Generalized Shallow Function}


$\mathrm{F}(\mathrm{x})=\sum_{\mathrm{i}=2}^{\mathrm{n} / 2}\left(\mathrm{x}_{2 \mathrm{i}-1}^{2}-\mathrm{x}_{2 \mathrm{i}}\right)^{2}+\left(1-\mathrm{x}_{2 \mathrm{i}-1}\right)^{2}$
$\mathrm{x}_{\mathrm{o}}=(-2, \ldots)^{\mathrm{T}}$

4. Nondiagonal Variant of Rosenbrock Function

$\mathrm{F}(\mathrm{x})=\sum_{\mathrm{i}=2}^{\mathrm{n} / 2}\left[100\left(\mathrm{x}_{1}-\mathrm{x}_{\mathrm{i}}^{2}\right)^{2}+\left(1-\mathrm{x}_{\mathrm{i}}\right)^{2}\right]$

$\mathrm{x}_{\mathrm{o}}=(-1, \ldots)^{\mathrm{T}}$

5. Generalized Cantrel Function

$\mathrm{F}(\mathrm{x})=\sum_{\mathrm{i}=1}^{\mathrm{n} / 4}\left[\left(\exp \left(\mathrm{x}_{4 \mathrm{i}-3}\right)-\mathrm{x}_{4 \mathrm{i}-2}\right)^{4}+100\left(\mathrm{x}_{4 \mathrm{i}-2}-\mathrm{x}_{4 \mathrm{i}-1}\right)^{6}+\left(\arctan \left(\mathrm{x}_{4 \mathrm{i}-1}-\mathrm{x}_{4 \mathrm{i}}\right)\right)^{4}+x_{4 i-3}\right]$

$\mathrm{x}_{\mathrm{o}}=(1,2,2,2, \ldots)^{\mathrm{T}}$

6. Generalized Miele Function

$\mathrm{F}(\mathrm{x})=\sum_{\mathrm{i}=1}^{\mathrm{n} / 4}\left[\exp \left(\mathrm{x}_{4 \mathrm{i}-3}-\mathrm{x}_{4 \mathrm{i}-1}\right)^{2}+100\left(\mathrm{x}_{4 \mathrm{i}-2}-\mathrm{x}_{4 \mathrm{i}-1}\right)^{6}+\left(\tan \left(\mathrm{x}_{4 \mathrm{i}-1}-\mathrm{x}_{4 \mathrm{i}}\right)\right)^{4}+x_{4 i-3}^{8}+\left(x_{4 i}-1\right)^{2}\right]$

$\mathrm{x}_{\mathrm{o}}=(1,2,2,2, \ldots)^{\mathrm{T}}$

7. Generalized Wood Function

$\mathrm{F}(\mathrm{x})=\sum_{\mathrm{i}=2}^{\mathrm{n} / 4}\left[100\left(\mathrm{x}_{4 \mathrm{i}-2}-\mathrm{x}_{4 \mathrm{i}-3}^{2}\right)^{2}+\left(1-\mathrm{x}_{4 \mathrm{i}-3}\right)^{2}+90\left(\mathrm{x}_{4 \mathrm{i}}-\mathrm{x}_{4 \mathrm{i}-1}^{2}\right)^{4}+\left(1-x_{4 i-1}^{2}\right)^{2}+10.1\right]$

$\mathrm{x}_{\mathrm{o}}=(-3,-1,-3,-1, \ldots)^{\mathrm{T}}$

8. Generalized Dixon Function

$\mathrm{F}(\mathrm{x})=\sum_{\mathrm{i}=1}^{\mathrm{n}}\left[\left(1-\mathrm{x}_{1}\right)^{2}+\left(1-\mathrm{x}_{\mathrm{n}}\right)^{2}+\sum_{\mathrm{i}=1}^{\mathrm{n}}\left(\mathrm{x}_{\mathrm{i}}^{2}-\mathrm{x}_{\mathrm{i}+1}\right)^{2}\right]$

$\mathrm{x}_{\mathrm{o}}=(-1, \ldots)^{\mathrm{T}}$

9. Generalized Rosenbrock Function

$\mathrm{F}(\mathrm{x})=\sum_{\mathrm{i}=2}^{\mathrm{n} / 2} 100\left(\mathrm{x}_{2 \mathrm{i}}-\mathrm{x}_{2 \mathrm{i}-1}^{2}\right)^{2}+\left(1-\mathrm{x}_{2 \mathrm{i}-1}\right)^{2}$

$\mathrm{x}_{\mathrm{o}}=(-1,2,1, \ldots)^{\mathrm{T}}$

\section{Beale Function}

$\mathrm{F}(\mathrm{x})=\left(1.5-\mathrm{x}_{1}\left(1-\mathrm{x}_{2}^{2}\right)\right)+\left(2.25-\mathrm{x}_{1}\left(1-\mathrm{x}_{2}^{2}\right)\right)^{2}+\left(2.625-\mathrm{x}_{1}\left(1-\mathrm{x}_{2}^{3}\right)\right)^{2}$

$\mathrm{x}_{\mathrm{o}}=(0,0)^{\mathrm{T}}$ 


\section{المصاد}

[1] Al-Baali, M. (1985). "Descent Property and Global Convergence of the Fletcher-Reeves Method with Inexact Line Search". IMA J. Numer. Anal., pp.121-124.

[2] Al-Bayati, A. Y. (1991). "A New Family of Self-scaling VM Algorithms for Un Constrained Optimization". J. of Educ. \& Sci. Vol(12), pp.25-54.

[3] Al-Bayati, A. Y. and Younis, M. S. (1995). "Imperfect Self-scaling VM Algorithms for Nonlinear Un Constrained Optimization". J. of Educ. \& Sci. Vol(23), pp.174-183.

[4] Dai, Y. H. (2001). "New Properties on A Nonlinear Conjugate Gradient Method". Numer. Math. 89. 83-98.

[5] Dai, Y. H. and Yuan, Y. (1996). "Convergence Properties of the Fletcher- Reeves Method”. IMA J. Numer. Anal., 16(2), pp.155-164.

[6] Dai, Y. H. and Yuan, Y. (1998). "Some Properties of A New Conjugate Gradient Method". In: Advanced in Nonlinear Programming, ed Y. Yuan (Kluwer Academic, Boston) pp.251-262.

[7] Dai, Y. H. and Yuan, Y. (1999). "A Nonlinear Conjugate Gradient Method with A Strong Global Convergence Property”. SIAM J. Optimization, 10(1), 177-182.

[8] Paul Kang-Hoh Phua, Weiguo FAN, Yuelin ZENG. (1997). "Parallel Algorithms for Large-Scale Nonlinear Optimization".

[9] Powell, M. J. D. (1977). "Non Convex Minimization Calculations and the Conjugate Gradient Method". In: Lecture Notes in Mathematics, 1066 (Springer, Berlin, 1984) pp.122-141.

[10] Touati-Ahmed, D. and Storey, C. "Efficient Hybrid Conjugate Techniques". Jota, 64(1990) 379-397. 